\title{
Experimental study of supersonic turbulent corner flow evolution in a low aspect ratio rectangular channel
}

\author{
Rohan R. Morajkar ${ }^{1}$, James F. Driscoll ${ }^{2}$ and Mirko Gamba ${ }^{3}$ \\ University of Michigan, Ann Arbor
}

\begin{abstract}
Supersonic turbulent flows in a rectangular duct are characterized by secondary flows in each of the four corners of the duct. These secondary flows comprise of two counter rotating vortices forcing the fluid towards the corner. The vortices are developed as a result of the Reynolds stress gradients which exist in each of the corners. Previous studies ${ }^{1,2}$ have primarily used intrusive techniques such as Preston/pitot tubes and hot wire in order to study the secondary flows in the corners. However use of these techniques would substantially modify the flow in low aspect ratio rectangular channels. Thus, there is a need for non-intrusive measurements to study the evolution of corner vortices in such flows. In this study we present the experimental results of stereo particle image velocimetry conducted on a Mach 2.75 flow in a low aspect ratio rectangular channel. Data is recorded at multiple cross-section planes and analyzed to study the formation of corner vortices and their effects on the structure of the mean flow field. Results show that the corner vortices are formed in the corner and then convect away from it. There is a significantly strong secondary flow associated with the corner vortices possibly causing open flow separation. The vortices significantly modify the local variation of the skin friction coefficient.
\end{abstract}

\section{Nomenclature}

$\mathrm{X}$

$\mathrm{x}, \mathrm{y}, \mathrm{z}=$ Derivative in respective direction

w $\quad=$ Quantity evaluated at the wall

$\infty \quad=$ Quantity evaluated in the free stream

Superscripts:

$+\quad=$ Inner wall scale

= Corrected using van Driest (1951) compressible scaling

\footnotetext{
${ }^{1}$ Graduate Student Research Assistant, Dept. of Aerospace Engineering, AIAA member.

${ }^{2}$ Arthur B. Modine Professor, Dept. of Aerospace Engineering, AIAA Fellow.

${ }^{3}$ Assistant Professor, Dept. of Aerospace Engineering, AIAA member.
} 


\section{Introduction}

Flows along a streamwise corner are quite common in aerospace applications. Such flows are commonly found along the wing-body junction of an aircraft and rectangular air intake ducts of aircrafts. These flows often give rise to secondary transverse flow perpendicular to the direction of the streamwise bulk flow. Secondary flows play a role in convection of mass, momentum, vorticity and energy from the primary flow into the corner and from the corner into the primary flow ${ }^{3}$. Due to this, the secondary flows often influence the structure of the primary and deviate it away from a canonical structure. This may reduce the effective area available for the flow inside the duct thereby affecting the performance of the aircraft or induce external separations along the wing-body junction causing loss of lift. Corner flows are characterized by regions of low momentum fluid near the junction of the walls forming the corner. These regions are particularly susceptible to separation especially in sensitive flows involving complex phenomena like shock-boundary layer interactions. It is thus required to understand the characteristics of such a flow. The aim of this work is to study supersonic turbulent corner flows that would exist in the air intake of a supersonic aircraft and attempt to understand the structure and the driving properties of the flow.

\section{Formation of Secondary Corner flows}

The fluid flow which exists at the intersection of two walls is characterized by secondary motion (motion due to velocity components normal to the principal flow direction). Ridha ${ }^{4}$ published a review article describing many of the theoretical studies of the corner flow boundary layer that have been conducted. Rubin ${ }^{5}$ initially developed an analytical method to describe the laminar corner flow. He divided the flow cross-section into 4 parts: boundary layers along the two walls forming the corner, the corner overlap between the boundary layers and the core flow. The terms of the Navier-Stokes (N-S) and boundary layer equations were then expanded into an infinite series and the constants matched up to second degree in order to match the values of various components of velocity that asymptote towards the core flow and in the boundary layer overlap region. He thus obtained a numerical solution describing the flow in corner region .

Prandtl in 1952 formally divided the secondary flows into two categories, also known as secondary flows of the first and second kinds which have been explained by Bradshaw ${ }^{6}$. Secondary flows of the first kind originate because of mean flow skewing, and have been relatively well understood. However, much is to be known about secondary flows of the second kind, which are a result of variations of Reynolds stresses ${ }^{3,4}$. The streamwise corner flows which exist at the intersection of two surfaces intersecting in the principal flow direction fall into the second kind.

Secondary flows are characterized by streamwise vortices which arise due to the production of vorticity by Reynolds stresses in the conservation of vorticity equation. The pair of streamwise vortices that would exist in ideal symmetric flow conditions is shown in Figure 1. Perkins ${ }^{7}$ explains the formation of these vorticities in his work. The (steady) $\mathrm{x}$-vorticity conservation equation taking into account the Reynolds stresses can be written as:

$$
U \frac{\partial \omega_{x}}{\partial x}+V \frac{\partial \omega_{x}}{\partial y}+W \frac{\partial \omega_{x}}{\partial z}=v \nabla^{2} \omega_{x}+\omega_{x} \frac{\partial U}{\partial x}+P 1+P 2+P 3+P 4
$$

Equation 1

where the terms on the left-hand-side represent the total convection of streamwise component (in $\mathrm{x}$ direction) of vorticity $\left(\omega_{\mathrm{x}}\right)$. The first two terms on the right-hand-side are the viscous diffusion and vortex stretching, respectively. The third term is:

$$
P 1=\omega_{y} \frac{\partial U}{\partial y}+\omega_{z} \frac{\partial U}{\partial z}
$$

Equation 2

and describes the production of streamwise vorticity by skewing of the mean flow field and thus represents the secondary flow of the first kind. The fourth terms is

$$
P 2=\frac{\partial}{\partial x}\left(\frac{\partial \overline{u^{\prime} v^{\prime}}}{\partial z}-\frac{\partial \overline{u^{\prime} w^{\prime}}}{\partial y}\right)
$$

Equation 3

and describes the formation of vorticity due to streamwise gradients of the primary Reynolds shear stress gradients. This term is often ignored from most of the studies due to assumption of zero pressure gradients; whether this term is important in flows with strong pressure gradients is currently not known. The fourth term in the vorticity equation is

$$
P 3=\frac{\partial^{2}}{\partial y \partial z}\left(\overline{v^{\prime 2}}-\overline{w^{\prime 2}}\right)
$$

Equation 4

and represents the formation of vorticity due to anisotropy in direct turbulent stresses. This term is believed to be the prominent quantity that leads to creation of streamwise vortices in a corner. Unlike the isotropic nature of the 
turbulence in the core flow, the kinetic energy is shared unequally between different directions in the corner region which leads $P 3$ to take non-zero values. The final term in the vorticity equation is

$$
P 4=\left(\frac{\partial^{2}}{\partial z^{2}}-\frac{\partial^{2}}{\partial y^{2}}\right) \overline{v^{\prime} w^{\prime}}
$$

Equation 5

and depicts the formation of streamwise vorticity due to gradients in the secondary stress $\overline{v w}$. Together P2, P3 and $\mathrm{P} 4$ represent the quantities responsible for the development and sustenance of the secondary flow of the second kind. These terms are important in corner flows.

Gessner $^{3}$ experimentally evaluated the terms in the energy and vorticity balance equations using pressure taps and hot wire techniques. His results suggest that the transverse secondary flow is initiated as a direct result of the primary turbulent stress gradients normal to the corner bisector and that the anisotropy of the stresses does not play a major role in the secondary flow generation as suggested by Perkins ${ }^{7}$. He suggests that the variations in the curvature of iso-contours of the axial velocity that occur in the flow undergoing laminar-to-turbulent transition result into turbulent stress gradients along the iso-contour which necessitate the development of secondary flow from the concave to the convex side of the iso-contour. Brundrett and Baines ${ }^{8}$ conducted experimental investigation using hot-wire in order to characterize regions of vorticity creation and diffusion. Their results suggested that the diffusion of vorticity peaks up near the wall while the production of vorticity is associated with the region near corner bisectors, however is zero on the corner bisector itself while being of opposing signs on either side of it. They suggest that it is due to this that the vortices have a tendency to convect towards the walls. The zone of peak vorticity production is characterized by a vortex on each side of the corner bisector with opposite signs. It was found that the vorticity production is independent of the Reynolds number, but the vortex diffusion is not. They suggested this to be the reason for the secondary flow to be pushed towards the wall as the Reynolds number is increased. Kornilov and Kharitonov ${ }^{9}$ studied the structure of the corner flows in asymmetric configurations featuring unequal boundary layer thicknesses on the walls forming corners. One of their most important findings was that the vortex located towards the thicker boundary layer is stronger and larger than that towards the thinner boundary layer.

The unstable nature of these flows have been reported by various stability studies conducted by Alizard et al. ${ }^{10}$, Zamir $^{11}$ and Balachandar \& Malik ${ }^{12}$. As a result of this unstable nature it is highly likely that any intrusive form of measurement would lead to a disturbed flow yielding false measurements especially in supersonic flow wherein the effect of shock-wave/boundary-layer interaction resulting from the probe can propagate upstream through the viscous region and change the characteristics of incoming flow as has been explained by Chapman et al. ${ }^{13}$ and reported by Kornilov ${ }^{14}$ amongst many others. Because of this, it is necessary to characterize the flow using the latest non-invasive experimental techniques, such as the particle image velocimetry (PIV) technique. Motivated by this observation, Park et al. ${ }^{15}$ conducted PIV measurements in the corner flow in a plane oriented along the corner bisector in the streamwise direction. They majorly focused on capturing the developing boundary layer on this plane. Their findings are in agreement with the theoretical studies in that the velocity distributions were found to follow self-similar profiles and that the corner velocity profiles correspond to the Blasius branch of the theoretical solutions. However in order to fully understand the secondary flows, it is necessary to obtain non-invasive high resolution measurements in the plane where such secondary flows are observed. This paper aims to fulfill this shortcoming.

\section{Experimental Setup}

\section{Wind tunnel setup}

The work presented in this article is a result of the experiments carried out at the Michigan Glass Wind Tunnel (GWT) facility. A schematic diagram of the configuration used in the study is shown in Figure 2. It is a low aspect ratio suction supersonic wind tunnel $57.2 \mathrm{~mm} \times 69.3 \mathrm{~mm}$ (2.25 in x $2.75 \mathrm{in})$ in cross-section currently configured to operate nominally at Mach 2.75 with stagnation pressure and temperature of $98.1 \mathrm{kPa}$ and $294 \mathrm{~K}$, respectively. The effective Mach number is approximately 2.72 . The tunnel is composed of a one-sided two-dimensional convergingdiverging nozzle followed by a constant area test section. This design was selected to produce an equilibrium flat plate boundary layer ${ }^{16}$ in an attempt to minimize pressure gradients history effects on the boundary layer developing on the bottom-wall (floor) of the wind tunnel. Since our previous work has focused on a 3D SBLI configuration and this work is an effort to better understand it, we use the same coordinate system of our previous SBLI work ${ }^{17,18,19}$. In particular, the origin of the coordinate system is centered at the location of the leading edge of the full-span $6^{\circ}$ shock generator wedge (which is about $481.5 \mathrm{~mm}$ downstream of the nozzle throat) used in our 3D SBLI work. However, in this work the shock generator has been removed. The unit Reynolds number of the flow is $8.9 \times 10^{6} / \mathrm{m}$ with an 
incoming boundary layer thickness ( $\delta$ ) of $10 \mathrm{~mm}$ measured at $x=75.5 \mathrm{~mm}$ and $y=28.6 \mathrm{~mm}$ in an empty tunnel ${ }^{16}$. The pressure gradient parameter defined as $\beta=\frac{\delta}{\rho U_{\infty}^{2}} \frac{d P}{d x}$ was calculated from side-wall static pressure measurements conducted in the empty tunnel at half the tunnel height. The value of this parameter was found to be $5 \times 10^{-4}$ for this experiment. A complete summary of the experimental conditions along with their respective uncertainties is provided in Table 1. Optical access to the test section is provided from both sides of the wind tunnel by glass windows that run along the whole length of the wind tunnel, including the nozzle throat region.

\section{2. $\quad$ Stereo Particle Image Velocimetry (SPIV)}

SPIV measurements are being performed. Two interline transfer CCD cameras (LaVision Flowmaster) recording at $3.33 \mathrm{~Hz}$ with a resolution of $1280 \times 1024$ pixel are used for the imaging. The cameras are placed in a stereoscopic, forward-scattering configuration oriented at $33^{\circ}$ with respect to the measurement plane. The cameras feature a minimum interframe time delay of about $500 \mathrm{~ns}$. The double-pulse illumination of the flow is provided by a pair of low repetition rate, frequency-doubled Nd:YAG lasers producing an output of $532 \mathrm{~nm}$ beam with a total energy of $200 \mathrm{~mJ} / \mathrm{pulse}$. The lasers are triggered at $10 \mathrm{~Hz}$ with a time delay of $650 \mathrm{~ns}$ in between the two pulses and pulse duration of $10 \mathrm{~ns}$. The delay is measured with a ThorLabs DT10A/M photodiode that has a $1 \mathrm{~ns}$ response time and a LeCroy Waverunner $6030350 \mathrm{MHz}$ digital oscilloscope and then ensured by adjusting the time lags between the trigger signals. In order to optimize the particle dropout and volumetric effects laser sheet thickness is set using expanding-collimating optics to approximately 4 times the out of plane displacement of a particle within the measurement domain as suggested by Adrian and Westerweel ${ }^{20}$. The beam width was measured to be $1.45 \pm 0.25 \mathrm{~mm}$ which is approximately 3.6 times the particle displacement (approx. $400 \mu \mathrm{m}$ ) in $650 \mathrm{~ns}$ at the free-stream speed.

Particle seeding of the flow was generated by a TDA-4B portable Laskin nozzle aerosol. The generator consists of an array of six Laskin nozzles that create poly-dispersed sub-micron particles using Poly-Alpha Olefin (PAO) oil with density of $819 \mathrm{~kg} / \mathrm{m}^{3}$.

The LaVision DaVis 8 software is used for the acquisition of the measurement and processing of the data. The three-component velocity fields are reduced from the particle images using Davis 8. A multi-pass with reducing interrogation window size is used. Two passes were conducted using an interrogation window size of 64x64 pixels with a $50 \%$ overlap. The final size of the interrogation windows after two further passes was $24 \times 24$ pixels with an overlap of 75\%, which corresponds to a projected physical size of about $0.47 \mathrm{~mm} \times 0.47 \mathrm{~mm}$ and a vector resolution of $0.11 \mathrm{~mm} \times 0.11 \mathrm{~mm}$ spanning a physical region of $17 \mathrm{~mm} \times 17 \mathrm{~mm}$. Post processing within multiple passes included deleting a vector if its correlation value was less than 0.8 as well as removing groups with less than 4 vectors. Post processing was also conducted once all the passes were completed by removing vectors with a peak ratio $(\mathrm{Q})$ less than 1.2. Laser sheets were oriented perpendicular to the flow, spanning a fraction the cross-section of the tunnel. Multiple such images (about 1000 instances at each measurement location) were recorded to construct distributions of various flow properties.

All of the SPIV data was pre-processed using PIVMAT which is a toolbox developed by F. Moisy for MATLAB and filtered when necessary using the smoothing-interpolating method developed by D. Garcia ${ }^{21}$ owing to the noisy nature of high resolution SPIV.

Measurements are performed on the lower right corner (as one looks downstream) of the duct cross-section at three streamwise direction: $-100 \mathrm{~mm},-50 \mathrm{~mm}$ and $0 \mathrm{~mm}$ upstream of the position where we have positioned the leading edge of a shock generator in our previous work ${ }^{17,22}$.

\section{Results and discussions}

\section{A. Primary Flow}

Figure 3a shows the streamwise velocity contours of the most upstream data-plane with the streamwise flow being out of the plane. It can be seen that the boundary layer thickness, defined by the $99 \%$ iso-contour line, is fairly uniform without any curvature indicating that the flow is fairly symmetric relative to the corner at this location. Also the close proximity of the sonic line to the side-wall indicates that the flow is majorly supersonic throughout the cross-section of the tunnel at $x=100 \mathrm{~mm}$. The sonic line was computed using the local speed and assuming a calorifically perfect gas with unit Prandtl number and a $T_{\infty} / T_{w}$ value of 0.37 for Mach 2.75 by interpolating the experimental values compiled by Spina et al. ${ }^{23}$

It can be seen from Figure $3 \mathrm{~b}$ (plane at $x=-50 \mathrm{~mm}$ ) that the side-wall $99 \%$ boundary layer thickness has started to thicken towards the bottom-wall before it merges with the bottom-wall boundary layer. On the contrary, the boundary layer on the bottom-wall remains fairly undisturbed. The sonic line now extends along the entire walls of 
the tunnel. Also a fair portion of the flow is subsonic at the corner. It can be observed that the subsonic flow is biased towards the side-wall rather than the bottom-wall, probably due to the one-sided nature of the nozzle.

Figure $3 \mathrm{c}$ is located at the origin of the co-ordinate system used here. At this location, we can observe that the structure of the primary flow has been significantly affected by the secondary corner flow. The sonic line has been deformed near the corner confirming to the low momentum bubble which now exists on the side-wall about $5 \mathrm{~mm}$ away from the corner. A similar weaker curvature in the sonic line can also be observed near the bottom-wall which peaks at about $y=15 \mathrm{~mm}$. At the same time, the sonic line can be seen penetrating towards the corner. This effect of curvature of iso-tach (constant velocity) lines can be observed till the boundary layer is approached. It can be observed that the side-wall boundary layer at $x=0 \mathrm{~mm}$ is similar to the side-wall boundary layer at $x=-50 \mathrm{~mm}$, however, the bottom-wall boundary layer has thickened and can be seen thickening further away from the corner contrary to the observation at the upstream planes.

\section{B. Secondary Flow}

Contour plots of the transverse (in-plane) velocity components are shown in Figure 4 and Figure 5. Although the streamwise velocity field at $x=-100 \mathrm{~mm}$ is fairly uniform, a study of the transverse velocity field reveals the presence of a weak secondary flow in proximity of the corner as is seen in Figure $4 \mathrm{a}$ and $4 \mathrm{~b}$ and Figure $5 \mathrm{a}$ and $5 \mathrm{~b}$. The $v$-component at these locations is directed towards the corner in the immediate vicinity of the corner and then reverses direction away from corner along the side-wall. At $x=0 \mathrm{~mm}$ (Figure $4 \mathrm{c}$ and Figure $5 \mathrm{c}$ ) the secondary flow is strongly developed: we can observe regions where the flow convects the higher momentum fluid from the core flow towards the corner and then away from the side-wall. This transport path causes the peculiar deformation of the primary flow structure seen in the previous section. This behavior was also documented by Gessner et al. ${ }^{1}$ although in our case the flow is not symmetric relative to the corner bisector.

\section{Wall normal gradients of streamwise velocity}

Streamwise shear stresses were computed from the mean streamwise velocity distributions and are shown in Figure 6 and Figure 7. Figure 6a and Figure 6b which show d $u / \mathrm{d} y$ at $x=-100 \mathrm{~mm}$ and $x=-50 \mathrm{~mm}$, are similar in structure. The shear layer near the side-wall is well defined in Figure 6a tapering as the corner is approached. In Figure $6 \mathrm{~b}$ we can see that the shear layer by the side-wall now extends further into the core flow with some bulging at $z=4 \mathrm{~mm}$. The corner flow is relatively well developed in Figure $6 \mathrm{c}$ and the shear zone now bulges into the core flow and conforms to the side-wall as the corner is approached. A prominent discontinuity in the shear layer by the wall is also seen around $z=5 \mathrm{~mm}$ possibly indicating an open flow separation ${ }^{24}$. Wu et al. ${ }^{24}$ define open separation as non-conformity of the shear layer to the bounding wall in the context of wall-bounded shear flow. Based on their definition, open separation is said to occur when the shear layer leaves the bouding wall causing, for example, a discontinuity in the regin of high shear near the wall. In this context, the wall-normal gradient of the streamwise velocity shown in Figure $6 \mathrm{c}$ show that the shear layer no longer conforms to the wall at about $\mathrm{z}=5 \mathrm{~mm}$, creating a gap in the region of high shear near the wall. From Figure 3c (vec fields) we can identify a region of flow upwelling at the location of shear layer gap. We therefore associate this flow feature to the flow separation as defined by Wu et

$\mathrm{al}^{24}$. This would be caused by the strong $v$-component of velocity as seen in Figure $4 \mathrm{c}$ which would cause fluid particles adjacent to the wall to upwell and no longer adhere to the wall causing flow separation.

Figure 7a and Figure $7 \mathrm{~b}$ show the distribution of $\mathrm{d} u / \mathrm{d} z$ at $x=-100 \mathrm{~mm}$ and $x=-50 \mathrm{~mm}$. In both these images the shear layer is well conformed to the bottom-wall, tapering towards the corner. From Figure $7 \mathrm{c}$ at $x=0 \mathrm{~mm}$ it can be seen that the well-developed corner flow squeezes the shear zone near the corner while it extends further into the core flow away from the corner.

\section{Corner vortices}

Because the flow in the corner is dominated by heavy shear and strain, traditional Cauchy Stoke's decomposition of motion into symmetric part (shear) and anti-symmetric (vorticity) is insufficient. The shear and strain mask the vorticity associated with vortex tubes and makes identifying the corner vortices difficult. Thus, a planar surrogate of Triple Decomposition of Motion (TDM) developed by $\mathrm{Kolar}^{25}$ was used to determine only the part of vorticity associated with rigid-body rotation $\left(\omega_{\mathrm{RR}}\right)$, which makes identifying the corner vortices clearer. Fields of rigid rotation vorticity obtained from the SPIV data are shown in Figure 8. Lack of a clear region of high $\omega_{\mathrm{RR}}$ at $x=-100 \mathrm{~mm}$ (see Figure 8a) indicates that the corner vortices are not well developed at this location. However, two weak patchy regions (possibly due to limitations of the current SPIV data set) of opposing vorticities are observed at the corner in Figure 8a which is perhaps the site of development of the vortices. A counter clockwise rotating region of negative vorticity is labeled as Vortex A while a clockwise rotating region of positive vorticity is labeled as Vortex B as shown in Figure $8 \mathrm{a}$. Figure $8 \mathrm{~b}(x=-50 \mathrm{~mm})$ shows a $\omega_{\mathrm{RR}}$ field with similar characteristics, 
however the signature of vortex B can be seen to have convected away from the corner along the bottom-wall. It is highly likely that the secondary corner flow has not fully developed at $x=-100 \mathrm{~mm}$ and $x=-50 \mathrm{~mm}$, and the discontinuous patches of positive and negative vorticities are caused by vortices being formed due to gradients in fluctuation quantities ( $P 3$ and $P 4)$. The corner secondary flow is relatively well developed in at $x=0 \mathrm{~mm}$ (Figure $8 c)$ : Vortex A is now clearly visible; it is more prominent, strong and lifted away from the bottom-wall, centered at about $z=5 \mathrm{~mm}$. However, a counter-rotating conjugate of vortex A is not prominent. The difference in relative sizes and strengths between vortex A and vortex B would be due to combined effect of low aspect ratio tunnel cross section and one-sided nature of the nozzle.

\section{E. Variation of skin friction coefficient $\left(C_{\mathrm{f}}\right)$}

Skin friction coefficient along the side and the bottom-wall was computed using the integral method of Mehdi and White ${ }^{26}$. The integral method is derived by simplifying the $2 \mathrm{D}$ boundary layer $\mathrm{x}$-momentum equation also considering the Reynolds stress gradients. The final expression of $C_{\mathrm{f}}$ is given as:

$$
C_{f}=4 \int_{\frac{n}{\delta}=0}^{\frac{n}{\delta}=1}\left(1-\frac{n}{\delta}\right) \frac{\partial \bar{U}}{\partial n} d\left(\frac{n}{\delta}\right)+2 \int_{\frac{n}{\delta}=0}^{\frac{n}{\delta}=1}\left(1-\frac{n}{\delta}\right)^{2}\left(-\frac{\partial^{2} \bar{U}}{\partial n^{2}}\right) d\left(\frac{n}{\delta}\right)
$$

Equation 6

where $n$ is the wall normal direction, $\delta$ is the $99 \%$ boundary layer thickness and $\bar{U}$ is the mean velocity tangential to the wall, which in our case would be $U+V$ along the bottom-wall and $U+W$ along the side-wall. The variation of $C_{f}$ along the bottom-wall at $x=-100 \mathrm{~mm}, x=-50 \mathrm{~mm}$ and $x=0 \mathrm{~mm}$ is shown in Figure 9a. The values of $u_{\tau}$ corresponding to $C_{f}$ on the bottom-wall away from the corner $(y=17 \mathrm{~mm})$ are $32 \mathrm{~m} / \mathrm{s}, 32 \mathrm{~m} / \mathrm{s}$ and $27 \mathrm{~m} / \mathrm{s}$ at $x=-100$ $\mathrm{mm},-50 \mathrm{~mm}$ and $0 \mathrm{~mm}$, respectively. These values match fairly well with the value reported by Lapsa and Dahm ${ }^{16}$ $\left(u_{\tau}=31 \mathrm{~m} / \mathrm{s}\right)$ on the centerline using the same experimental setup but a different approach. The general trend of increase of $C_{f}$ away from the corner is in agreement with Davis et al. ${ }^{1}$, however the rest of the deviations may possibly be from the asymmetric nature of the experimental geometry. The red curve in Figure 9a represents the $C_{f}$ variation at $x=-100 \mathrm{~mm}$.

The effect of variation of $C_{f}$ due to longitudinal vortices imbedded in boundary layer was studied in detail by Shabaka et al. ${ }^{27}$. It was found that the regions where the velocity vectors point away from the wall lead to a lower $C_{f}$ while those with vectors pointing towards the wall lead to higher value of $C_{f}$ due to convection of lower momentum fluid away from and convection of higher momentum fluid towards the wall respectively. In accordance to this explanation it can be seen that the rate of growth of $C_{f}$ with respect to $y$ suddenly increases at about $y=6 \mathrm{~mm}$ which is the region where vortex B is developing (refer Figure 8a) and would convect the higher momentum fluid from the core flow towards the bottom-wall, which would increase the value of $\mathrm{d} u / \mathrm{d} z$ at $y=6 \mathrm{~mm}$. Vortex B would have a tendency to cause an upwelling at around $y=10 \mathrm{~mm}$ convecting the low momentum fluid from the corner towards the core flow, thus reducing $\mathrm{d} u / \mathrm{d} z$ and hence $C_{f}$. This reduction in $C_{f}$ is observed in Figure $9 \mathrm{a}$. The lower rate of increase of $C_{f}(x=-50 \mathrm{~mm})$ may be attributed to strengthening vortex A or relative weakening of vortex B causing the effects of vortex A to dominate over vortex B which is observed in the magnitudes of Figure 8b. However, the location of strong increase of $C_{f}(y=8 \mathrm{~mm})$ and the location of its decrease $(y=11 \mathrm{~mm})$ correspond well with the zone of development of vortex A. It can be seen from Figure 9a that the curve of $C_{f}$ at $x=0 \mathrm{~mm}$ (well developed corner secondary flow) is lower than those at $x=-100 \mathrm{~mm}$ and $x=-50 \mathrm{~mm}$, which would be due to the dominance of vortex A over vortex B. The strong peak in $C_{f}(x=0 \mathrm{~mm})$ at about $y=5 \mathrm{~mm}$ can be attributed to vortex A convecting higher momentum fluid from away from the corners and walls towards the bottom-wall which is evident from the in plane unit vectors.

Variation of $C_{f}$ with $z$ is shown in Figure 9b. It can be seen from $C_{f}(x=-100 \mathrm{~mm})$ and $C_{f}(x=-50 \mathrm{~mm})$ that there are multiple minima in the region from the corner to $z=3 \mathrm{~mm}$. These may possibly correspond to likely sites of development of vortex A which convects the lower velocity fluid from near the wall causing minima. We can see from the curve corresponding to variation of $C_{f}$ with respect to $z$ in Figure 9b, that there exists a maxima at $z=2 \mathrm{~mm}$ and a minima at $z=3 \mathrm{~mm}$. This is caused by vortex B convecting high momentum fluid from the free stream towards the corner which is incident at $z=2 \mathrm{~mm}$ as seen in Figure 8c. This increases the $\mathrm{d} u / \mathrm{d} y$ thereby increasing $C_{f}$. Similarly vortex B appears to cause a positive $v$ velocity starting at $z=3 \mathrm{~mm}$ which would convect the lower velocity corner fluid towards the core decreasing $\mathrm{d} u / \mathrm{d} y$ and causing a minima at $z=3 \mathrm{~mm}$.

It can thus be said that the corner vortices play an important role towards the distribution of $C_{f}$ around the corner.

\section{F. Effect of secondary flow on the primary flow}

Profiles of the streamwise velocity at $x=-100 \mathrm{~mm}$ normal to the bottom-wall and side-wall are shown in Figure 10a and Figure 11a respectively. The velocity profiles do not show any visible inflection points and seem to be 
similar to that of an attached flow (see $y=5 \mathrm{~mm}$ and $z=5 \mathrm{~mm}$ in Figure 10a and Figure 11a). At the downstream location $x=-50 \mathrm{~mm}$, it can be seen from Figure 10b and Figure $11 \mathrm{~b}$ that the velocity profile now sports an inflection point near the corner $(y=5 \mathrm{~mm}$ and $z=5 \mathrm{~mm}$ ) while the flow at $y=10 \mathrm{~mm} \& 15 \mathrm{~mm}$ and $z=10 \mathrm{~mm} \& 15 \mathrm{~mm}$ still continues to resemble that of a generic flow over a flat plate. Influence of the secondary corner flows can be seen in Figure 10c. The streamwise velocity profile at $y=5 \mathrm{~mm}$ is seen to have a minimum point at about $z=5 \mathrm{~mm}$ which corresponds to the corner vortex A drawing the low momentum fluid from the corner at this location (refer to Figure $8 \mathrm{c}$ for a description of the vortex system). Vortex $\mathrm{A}$ is also responsible for convecting the higher momentum free stream fluid towards the bottom-wall at about $y=10 \mathrm{~mm}$ (see in plane unit vectors in Figure $3 \mathrm{c}$ ) which causes an increase in the shape factor at $y=10 \mathrm{~mm}$ as seen in Figure 10c. The spanwise variation in streamwise velocity at $x$ $=0 \mathrm{~mm}$ is shown in Figure 11c. The velocity structure observed at this location is similar to that observed at $x=-50$ $\mathrm{mm}$ in Figure 10b, however more pronounced.

Velocity profiles in inner wall scaling at a spanwise distance of $17 \mathrm{~mm}$ and $12 \mathrm{~mm}$ from the corner is shown in Figure 12 and Figure 13, respectively. The shear velocity at the wall was obtained from a measure of the skin friction coefficient $C_{f}$ estimated from the current measurements using the method outlined in section IV E. It is seen from Figure 12a that the boundary layer velocity profile follows the theoretical log-law curve (Cole's Wake law with van Driest's compressible correction) at $x=-100 \mathrm{~mm}$; strong corner effects are not observed at this location. The data plane at $x=-50 \mathrm{~mm}$ is shown in Figure 12b. It can be seen that the velocity profile is fuller compared to the theoretical curve at $y=17 \mathrm{~mm}$, this fullness increases as the corner is approached which can be seen by comparing Figure 12b (top) and Figure 13b (top) which correspond to $y=12 \mathrm{~mm}$. This is due to the net downward flow generated by the stronger vortex A compared to vortex $\mathrm{B}$, which brings the higher momentum fluid from the core flow towards the wall. The velocity profiles at $z=17 \mathrm{~mm}$ and $z=12 \mathrm{~mm}$ more or less follow the theoretical curve. As seen from the Figure 12c (top), the velocity profile is further above the Cole's wake curve since the corner vortices are well developed at $x=0 \mathrm{~mm}$ and there is a greater convection of the higher momentum core flow towards the bottom-wall. In Figure 13c (top), it is observed that the fullness has weakened due to the presence of vortex B at about $y=12 \mathrm{~mm}$ (see Figure 8c) which counters the net downward flow at this location. Departure from the theoretical behavior around the logarithmic region is consistently observed in Figure 12c (bottom) and Figure $13 \mathrm{c}$ (bottom) which corresponds to the location effected by a developed vortex A. This may be due to the convection of low momentum fluid from near the side-wall towards the core flow due to vortex A causing a momentum defect in the logarithmic region. The velocity profile was observed to not conform to the log law as corner was approached within $5 \mathrm{~mm}$. It was observed to lie above the theoretical curve indicating a net influx of high momentum from the core flow.

\section{G. Reynolds Stress distributions}

As per Gessner ${ }^{3}$ and Perkins ${ }^{7}$, the transverse components of Reynolds stresses play a key role in development of the streamwise vorticity, particularly the quantities P3 and P4 as described by Equation 4 and Equation 5. Figure 14 shows the iso-contour levels of the Reynolds stress $\overline{v^{\prime 2}}$ at the three data locations. It can be seen that the centers of vortices in Figure 8 lie very well within the zones of high $\overline{v^{\prime 2}}$ shear. The iso-contour distribution of Reynolds stress $\overline{w^{\prime 2}}$ is shown in Figure 15. It can be observed that $\overline{w^{\prime 2}}$ spans a lesser area and is weaker than $\overline{v^{\prime 2}}$ as seen in Figure 14. The distribution of $\overline{v^{\prime 2}}-\overline{w^{\prime 2}}$ which is one of the parameters governing the formation of corner vortices is shown in Figure 16. It can be seen from Figure 16a and $16 \mathrm{~b}$ that most of the vorticity formation due to $\overline{v^{\prime 2}}-\overline{w^{\prime 2}}$ takes place near the wall and corner. Once vortex A is fully developed, variation in $\overline{v^{\prime 2}}-\overline{w^{\prime 2}}$ away from the corner to near the location of vortex $\mathrm{A}$ as is observed in Figure 16c. However, there is no strong variation in $\overline{v^{\prime 2}}-\overline{w^{\prime 2}}$ on the bottom-wall, which would mean that there is comparatively less vorticity production on the bottom-wall. The

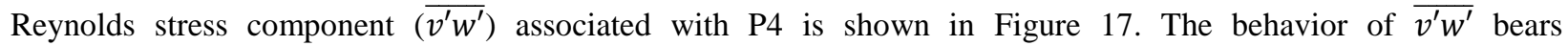
resemblance to that of $\overline{v^{\prime 2}}-\overline{w^{\prime 2}}$. The variation in $\overline{v^{\prime} w^{\prime}}$ is high near walls and corner where the vortex A resides in Figure 17a and 17b. In Figure 17c where the vortex A is lifted, heavy variation (change of signs) is spotted near the location of vortex A. However, again no strong variation is observed along the corner bottom-wall to indicate the formation of the counter vortex to A. Comparing the magnitudes in Figure 14, Figure 15, Figure 16 and Figure 17, it can be seen that the magnitudes of $\overline{v^{\prime 2}}, \overline{w^{\prime 2}}$ and $\overline{v^{\prime 2}}-\overline{w^{\prime 2}}$ are consistently at least an order of magnitude larger than $\overline{v^{\prime} w^{\prime}}$ and so is its variation over same distance. It can thus be estimated that the contribution of $\mathrm{P} 3$ would be larger than P4 which would be in agreement with the findings of Brundrett and Baines ${ }^{8}$. A computation of P3 and P4 was attempted, however the current data proved noisy due to the limitations of the current SPIV dataset. 


\section{Conclusions}

The supersonic turbulent corner flow was investigated in a low aspect ratio channel using stereo PIV. We considered the supersonic duct flow generated in our Mach 2.75 supersonic wind tunnel. We conducted the stereo PIV measurements over three planes orthogonal to the main flow direction. Measurements targeted the corner region to capture its flow evolution within the boundary layer. The three measurements planes were located well downstream of the end of the diverging portion of the nozzle, and are spaced by several boundary layer thicknesses. The resulting measurements were used to construct ensemble-average representation of the three velocity components, Reynolds stress components, and boundary layer profiles. Spanwise and wall-normal distributions of the skin friction coefficient were also estimated from the velocity profiles using the integral method of Mehdi and White $^{26}$. The data collected thus for in our configuration suggests the following conclusions:

- No form of symmetry was observed in the corner region for the given configuration of one-sided nozzle and low aspect ratio channel. A well-developed corner vortex was observed only on the third measurement plane after sufficient flow development has occurred. The flow at the corner itself was dominated by a single dominant strong vortex (toward the side-wall side of the corner) rather than by a vortex pair as other studies have found ${ }^{1-3,8}$. The corresponding second vortex appeared to be significantly displaced from the corner vertex itself. There is possibly an open flow separation once the corner vortex near the side-wall is well developed.

- The flowfield resulting from the formation of the dominant corner vortex near the side-wall is consistent with the existence of an open flow separation where flow reversal does not occur, but shear layer detachment from the wall exists as the flow approaching the corner region merge with the corner vortex as per the definition by $\mathrm{Wu}$ et al. ${ }^{24}$

- The corner vortex tends to decrease the value of the coefficient of friction in regions it draw the lower momentum fluid away from the wall and increase the coefficient of friction in regions where the higher momentum fluid from the core flow is convected towards the wall. This is consistent with skin friction measurements in boundary layers dominated by vortices ${ }^{27}$.

- Regions of large Reynolds stress components consistenly originate from the corner region itself (i.e., the corner vertex), and they evolve by moving toward the core flow preferentially along the side-wall rather then bottom-wall. As a consequence, we deduce that the regions of production of vorticity due to spatial variations of Reynolds stress gradients are preferentially located near the corner vortex center on the sidewall. As a result, the major contribution to vorticity production comes from the side-wall in the given experimental setup.

- The diagonal (in-plane) components of the Reynolds stress tensor are significant larger in magnitude than the corresponding $\overline{v^{\prime} w^{\prime}}$ component; their magnitude increase as the corner flow evolves along the duct.

- The velocity profiles (inner scale normalized) show fuller profiles in the outer wake region when the wallnormal velocity vectors point towards the wall, while they show a defect region in the logarithmic region when the wall-normal vectors point away from the wall, indicating momentum transport (upwell/downwell motion) due to the corner vortices.

\section{References:}

Davis, D. O., and Gessner, F. B., "Further Experiments on Supersonic Turbulent Flow Development in a Square Duct," AIAA JOURNAL, vol. 27, 1989, pp. 1023-1030.

Gessner, F. B., Eppich, H. M., and Lund, E. G., "Reynolds Number effects on near wall structure of turbulent flow along a streamwise corner," Near-Wall Turbulent Flows, 1993, pp. 965-975. 
Gessner, F. B., "The origin of secondary flow in turbulent flow along a corner," Journal of Fluid Mechanics, vol. 58, 1972, pp. 1-25.

Ridha, A., "Flow along streamwise corners revisited," Journal of Fluid Mechanics, vol. 476, Mar. 2003, pp. 223-265.

Rubin, S. G., "Incompressible flow along a corner," Journal of Fluid Mechanics, vol. 26, Mar. 1966, p. 97.

Bradshaw, P., "Turbulent Secondary Flows," Annual Review of Fluid Mechanics, vol. 19, Jan. 1987, pp. 53-74.

Perkins, H. J., "The formation of streamwise vorticity in turbulent flow," Journal of Fluid Mechanics, vol. 44, 1970, pp. 721-740.

Brundrett, E., and Baines, W. D., "The production and diffusion of vorticity in duct flow," Journal of Fluid Mechanics, vol. 19, 1963, pp. 375- 394.

Kornilov, V. I., and Kharitonov, a. M., "Investigation of the structure of turbulent flows in streamwise asymmetric corner configurations," Experiments in Fluids, vol. 2, 1984, pp. 205-212.

Alizard, F., Robinet, J.-C., and Rist, U., "Sensitivity analysis of a streamwise corner flow," Physics of Fluids, vol. 22, 2010, p. 014103.

Zamir, M., "Similarity and stability of the laminar boundary layer in a streamwise corner," Proceedings of the Royal Society: Mathematical, Physical and Engineering Sciences, vol. 377, 1981, pp. 269-288.

Balachandar, S., and Malik, M. R., "Inviscid instability of streamwise corner flow," Journal of Fluid Mechanics, vol. 282, Apr. 2006, pp. 187-201.

Chapman, D. R., Kuehn, D. M., and Larson, H. K., "Investigation of separated flows in supersonic and subsonic streams with emphasis on the effect of transition," NACA Technical Report 1356, NACA, 1957, pp. 421-460.

4 Kornilov, V. I., "Correlation of the separation region length in shock wave / channel boundary layer interaction," vol. 23, 1997.

Park, D. H., Park, S. O., Kwon, K. J., and Shim, H. J., "Particle Image Velocimetry Measurement of Laminar Boundary Layer in a Streamwise Corner," AIAA Journal, vol. 50, Apr. 2012, pp. 811-817.

Lapsa, A. P., and Dahm, W. J. A., "Stereo particle image velocimetry of nonequilibrium turbulence relaxation in a supersonic boundary layer," Experiments in Fluids, vol. 50, Jun. 2010, pp. 89-108.

Morajkar, R., Klomparens, R., Eagle, E., Driscoll, J., and Gamba, M., "Flow Separation Associated with 3 - D Shock - Boundary Layer Interaction (SBLI)," Harbor Town: American Institute of Aeronautics and Astronautics, 2014, pp. 1-13. 
Eagle, W. E., Driscoll, J. F., and Benek, J. A., "3-D Inlet Shock-Boundary Layer Interactions - PIV Database for the Second SBLI Workshop," 30th AIAA Applied Aerodynamics Conference, New Orleans, LA: American Institute of Aeronautics and Astronautics, 2012.

Eagle, W. E., Driscoll, J. F., and Benek, J. A., "Experimental Investigation of Corner Flows in Rectangular Supersonic Inlets with 3D Shock-Boundary Layer Effects," 49th AIAA Aerospace Sciences Meeting including the New Horizons Forum and Aerospace Exposition, Orlando, Florida: American Institute of Aeronautics and Astronautics, 2011, pp. 1-11.

Adrian, R. J., and Westerweel, J., Particle Image Velocimetry, Cambridge University Press, 2010.

Garcia, D., "Robust smoothing of gridded data in one and higher dimensions with missing values," Computational Statistics \& Data Analysis, vol. 54, Apr. 2010, pp. 1167-1178.

Morajkar, R. R., Klomparens, R. L., Eagle, W. E., Driscoll, J. F., Gamba, M., Wright, A., Air, P., and Base, F., "Relationship Between Intermittent Separation and Vortex Structure in a Low - Aspect Ratio 3D Shock Wave - Boundary Layer Interaction," Submitted to AIAA Journal.

Spina E., Smits A., R. S., "The physics of supersonic turbulent boundary layers," Annual Review of Fluid Mechanics, vol. 26, 1994, pp. 287-319.

Wu, J. Z., Tramel, R. W., Zhu, F. L., and Yin, X. Y., "A vorticity dynamics theory of threedimensional flow separation," Physics of Fluids, vol. 12, 2000, p. 1932.

Kolář, V., "Vortex identification: New requirements and limitations," International Journal of Heat and Fluid Flow, vol. 28, Aug. 2007, pp. 638-652. experimental data," Experiments in Fluids, vol. 50, May 2010, pp. 43-51. turbulent boundary layers . Part 1 . Single vortex," Journal of Fluid Mechanics, vol. 155, 1985, pp. 37-57. 


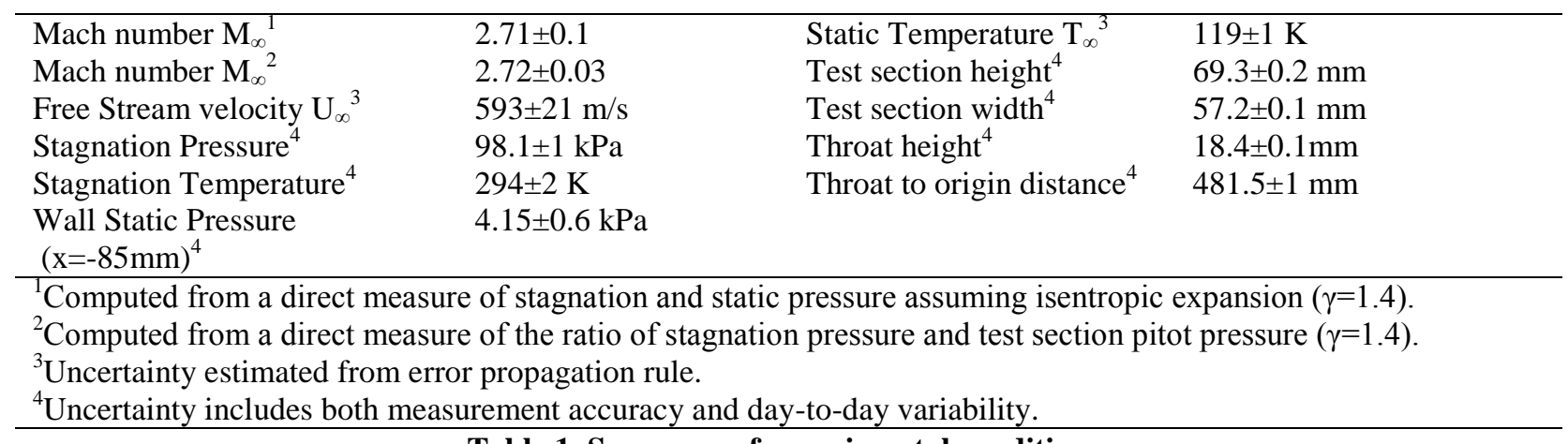

Table 1. Summary of experimental conditions.

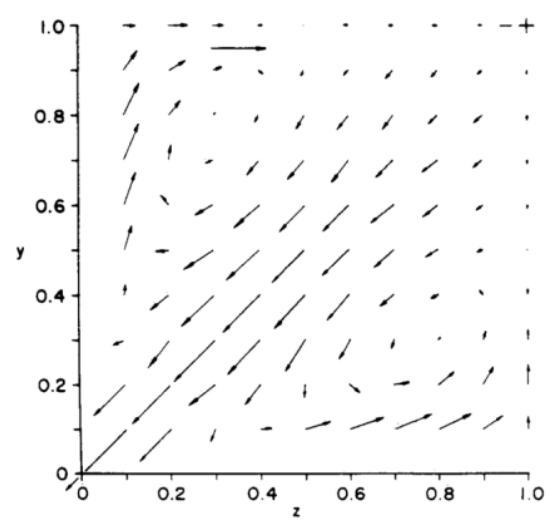

Figure 1. Corner vortices that would exist in an ideal symmetric configuration, net flow out of the plane, fron Davis et al. ${ }^{1}$ 


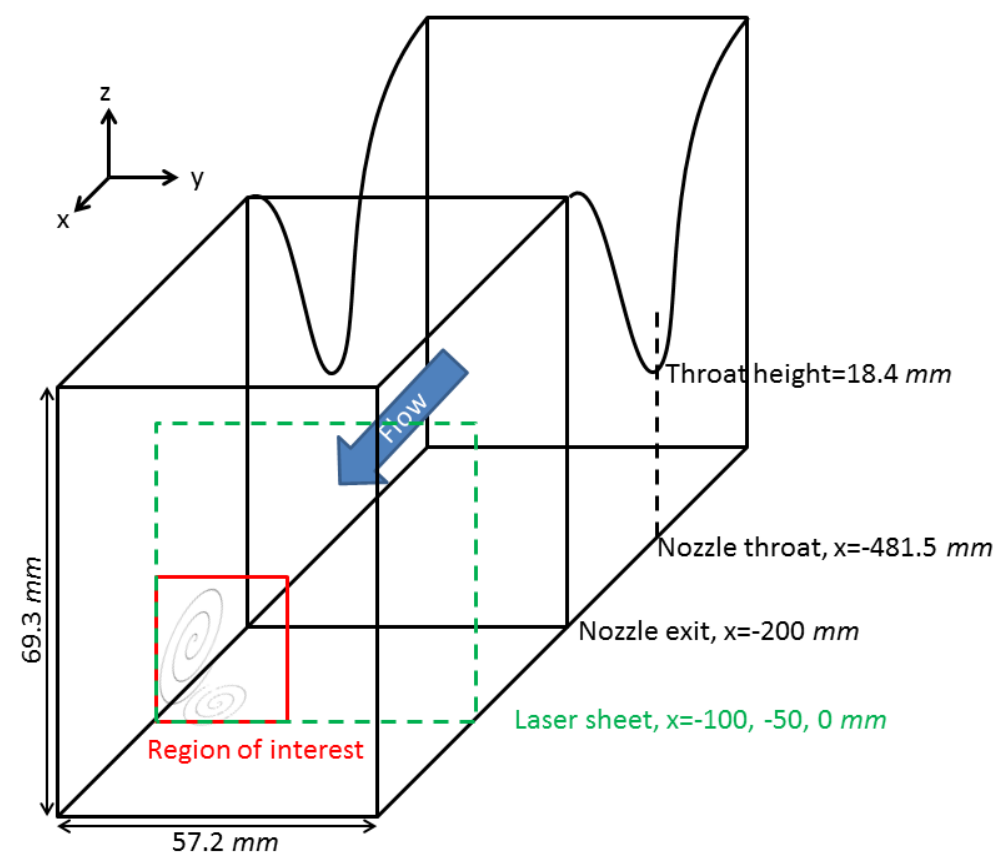

Figure 2. Wind Tunnel schematic (Not to scale)

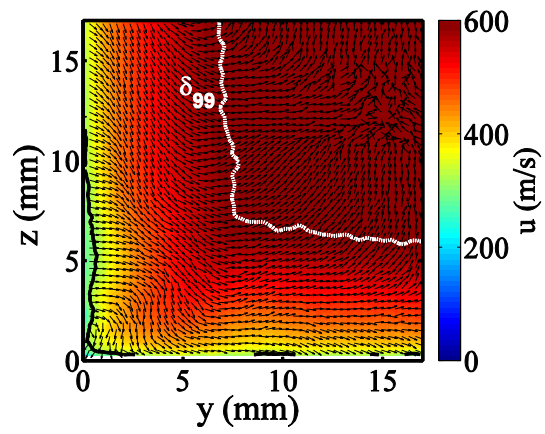

(a)

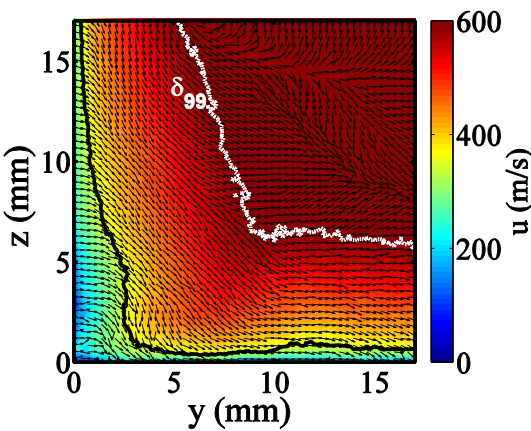

(b)

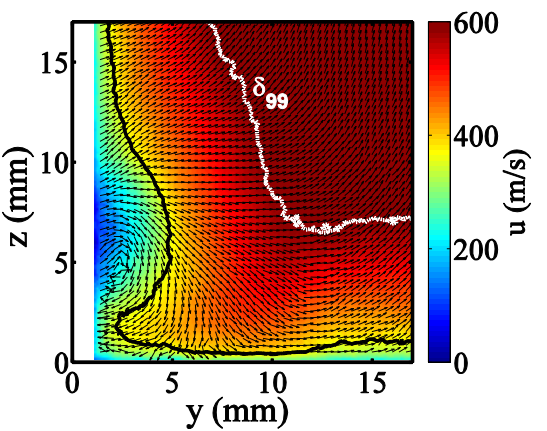

(c)

Figure 3. Average streamwise $U$ velocities overlapped with in plane unit vectors, the sonic line (black) and 99\% $U_{\infty}$ boundary layer (white) at (a) $x=-100 \mathrm{~mm}$, (b) $x=-50 \mathrm{~mm}$ and (c) $x=0 \mathrm{~mm}$. Every third vector is shown for clarity. 


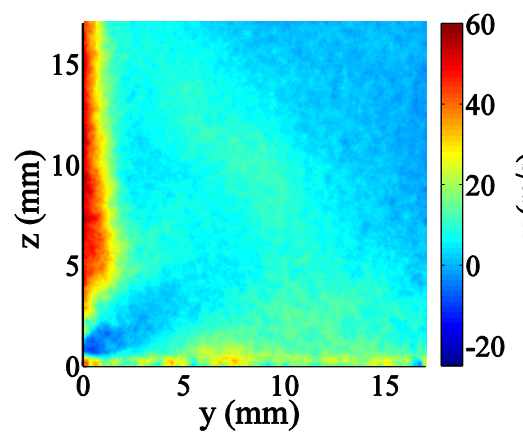

(a)

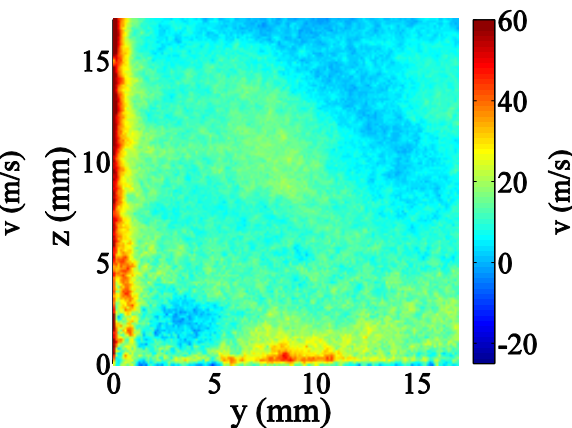

(b)

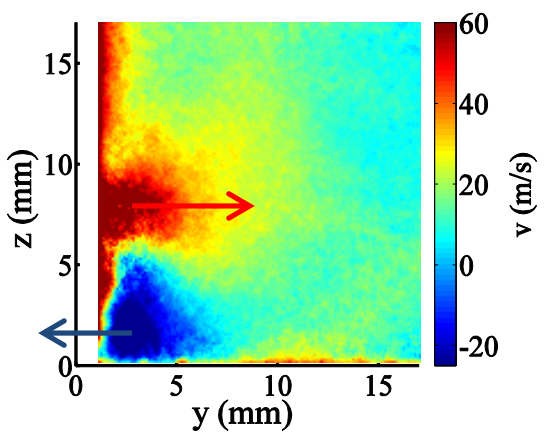

(c)

Figure 4. Average $v$ velocities at (a) $x=-100 \mathrm{~mm}$, (b) $x=-50 \mathrm{~mm}$ and (c) $x=0 \mathrm{~mm}$.

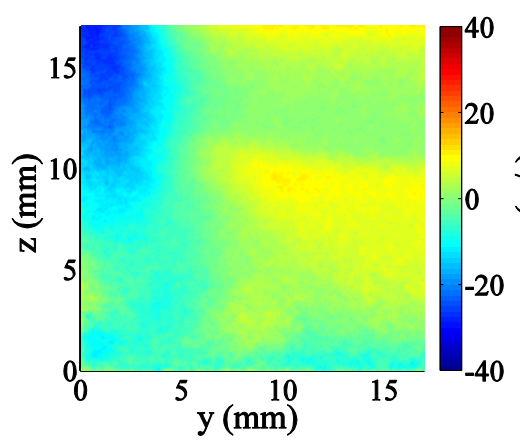

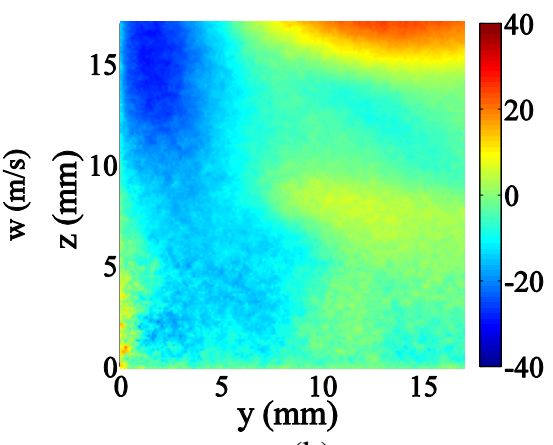

(b)

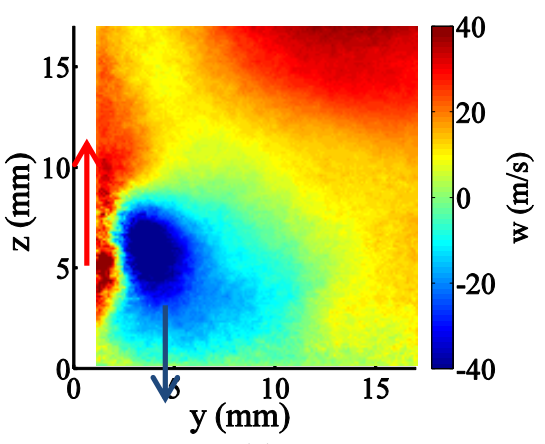

(c)

Figure 5. Average $w$ velocities at (a) $x=-100 \mathrm{~mm}$, (b) $x=-50 \mathrm{~mm}$ and (c) $x=0 \mathrm{~mm}$.

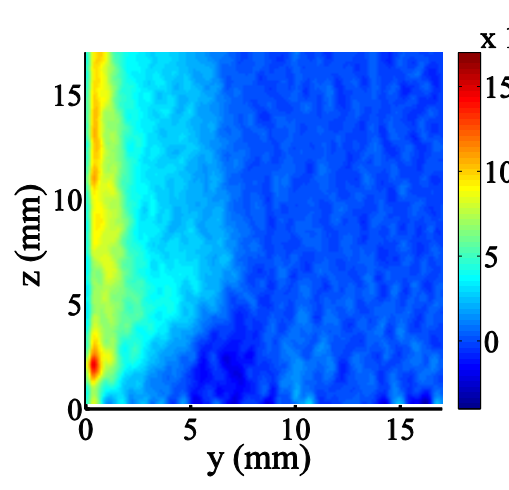

(a)

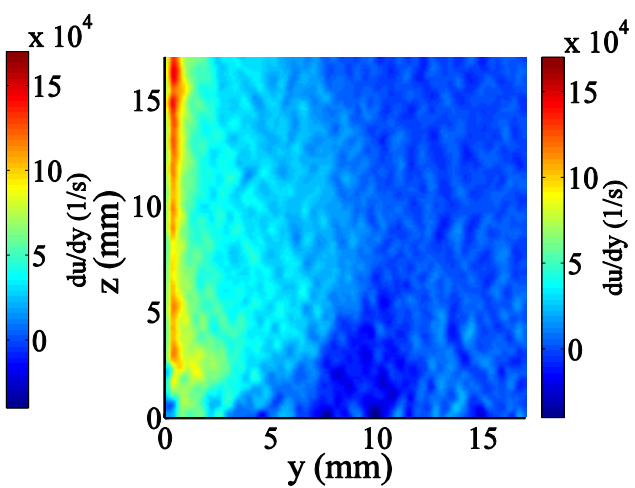

(b)

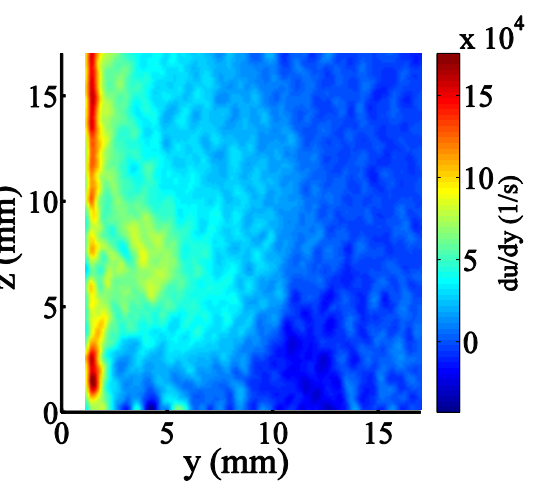

(c)

Figure 6. Average velocity gradient (du/dy) at (a) $x=-100 \mathrm{~mm}$, (b) $x=-50 \mathrm{~mm}$ and (c) $x=0 \mathrm{~mm}$. 


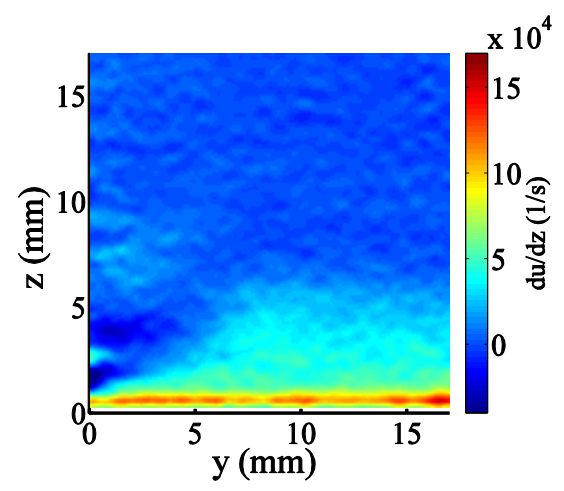

(a)

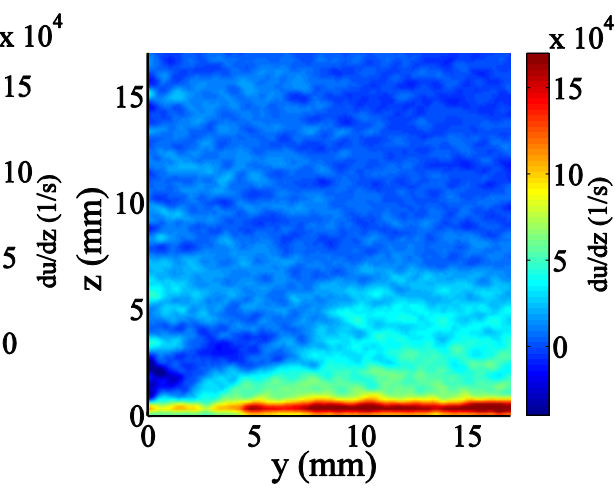

(b)

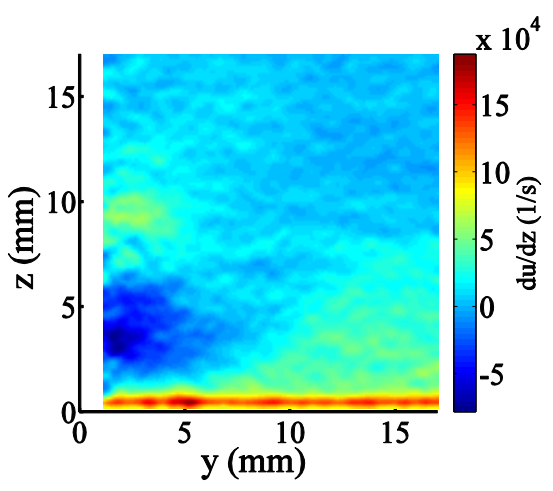

(c)

Figure 7. Average velocity gradient (du/dz) at (a) $x=-100 \mathrm{~mm}$, (b) $x=-50 \mathrm{~mm}$ and (c) $x=0 \mathrm{~mm}$.

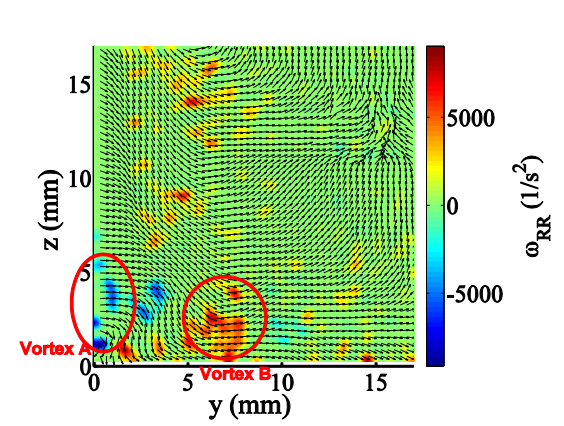

(a)

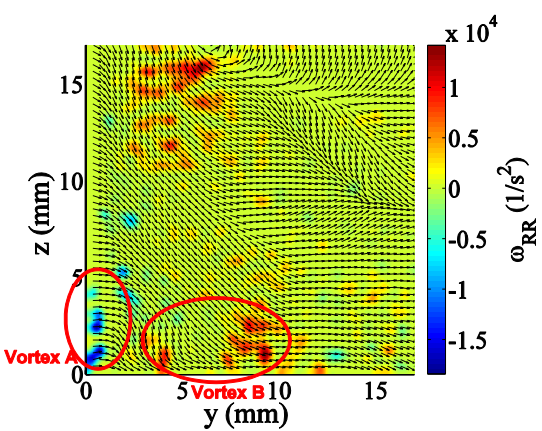

(b)

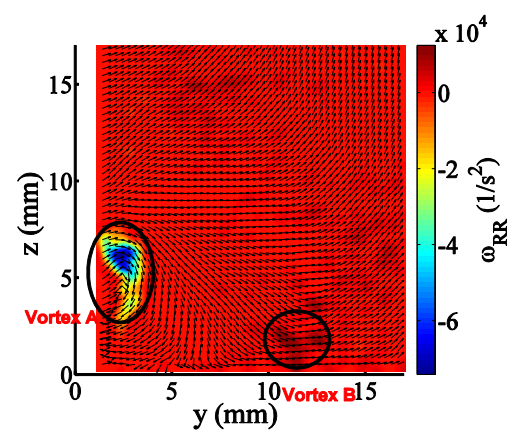

(c)

Figure 8. Rigid rotation $\mathrm{x}$-vorticity as obtained from TDM at (a) $x=-100 \mathrm{~mm}$, (b) $x=-50 \mathrm{~mm}$ and (c) $x=0$ mm.

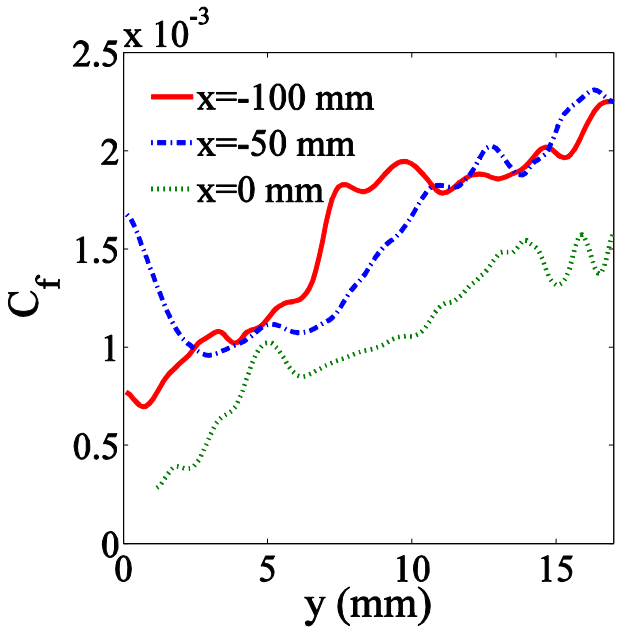

(a)

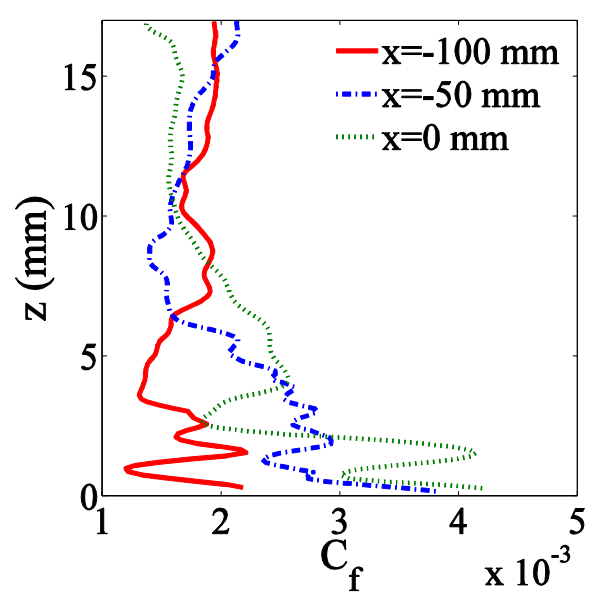

(b)

Figure 9. Variation of skin friction coefficient along (a) the bottom-wall and (b) the side-wall 


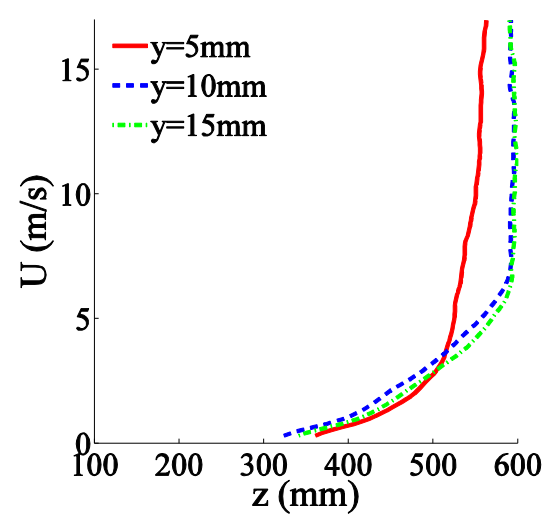

(a)

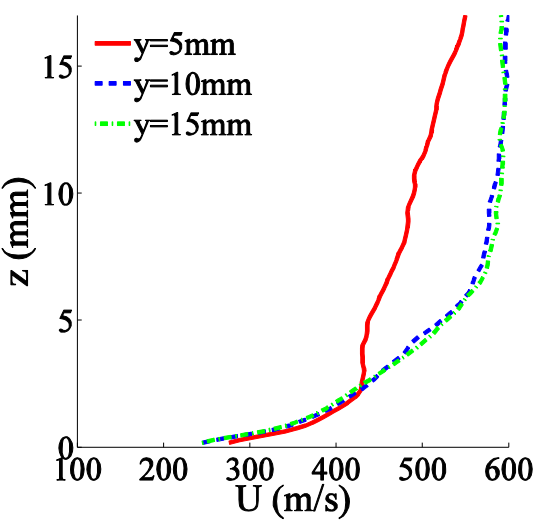

(b)

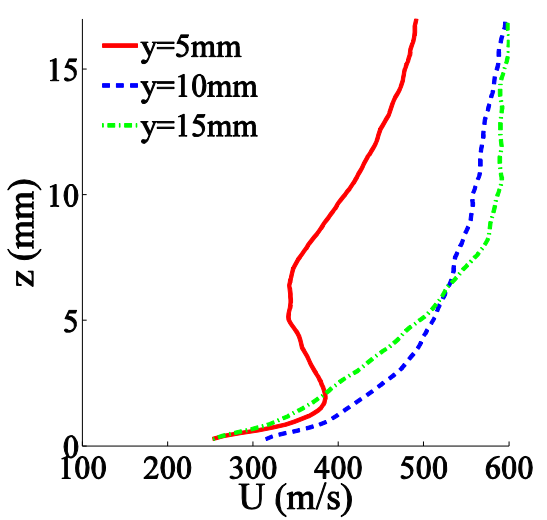

(c)

Figure 10. Average streamwise velocity variation with respect to $z$ at (a) $x=-100 \mathrm{~mm},(\mathrm{~b}) x=-50 \mathrm{~mm}$ and

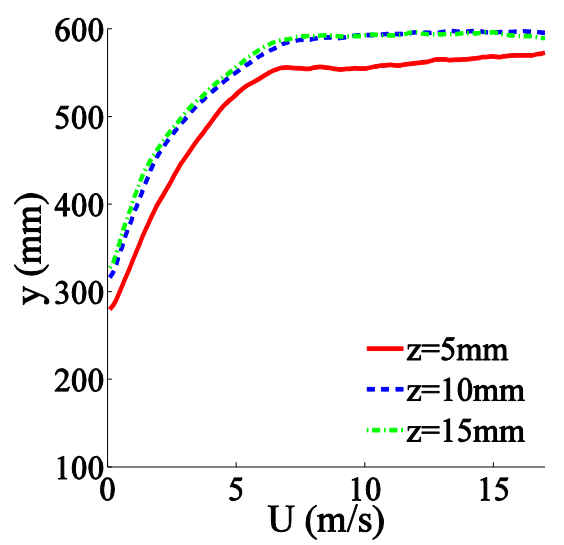

(a)

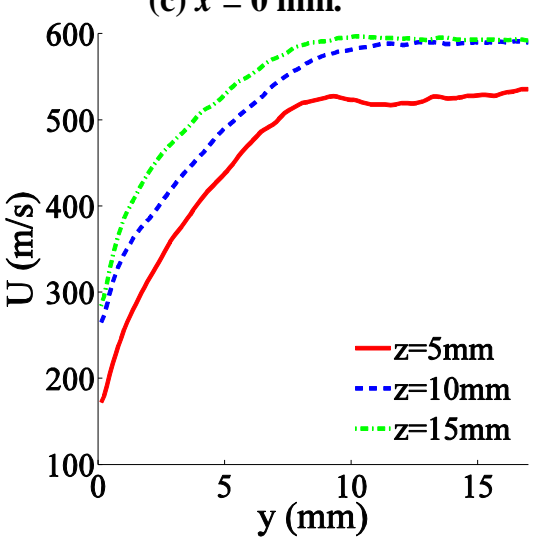

(b)

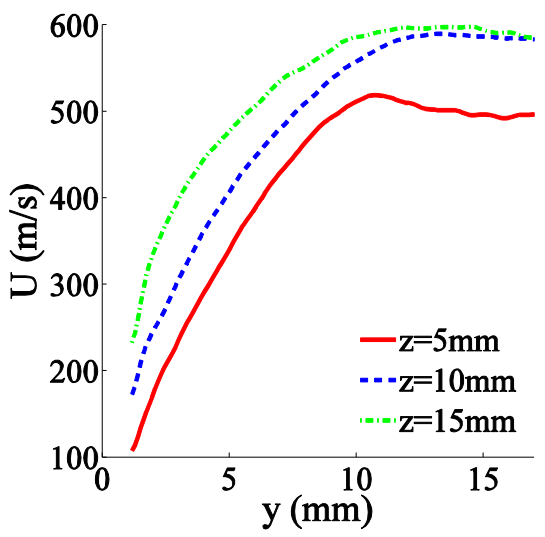

(c)

Figure 11. Average streamwise velocity variation with respect to $y$ at (a) $x=-100 \mathrm{~mm}$, (b) $x=-50 \mathrm{~mm}$ and (c) $x=0 \mathrm{~mm}$.
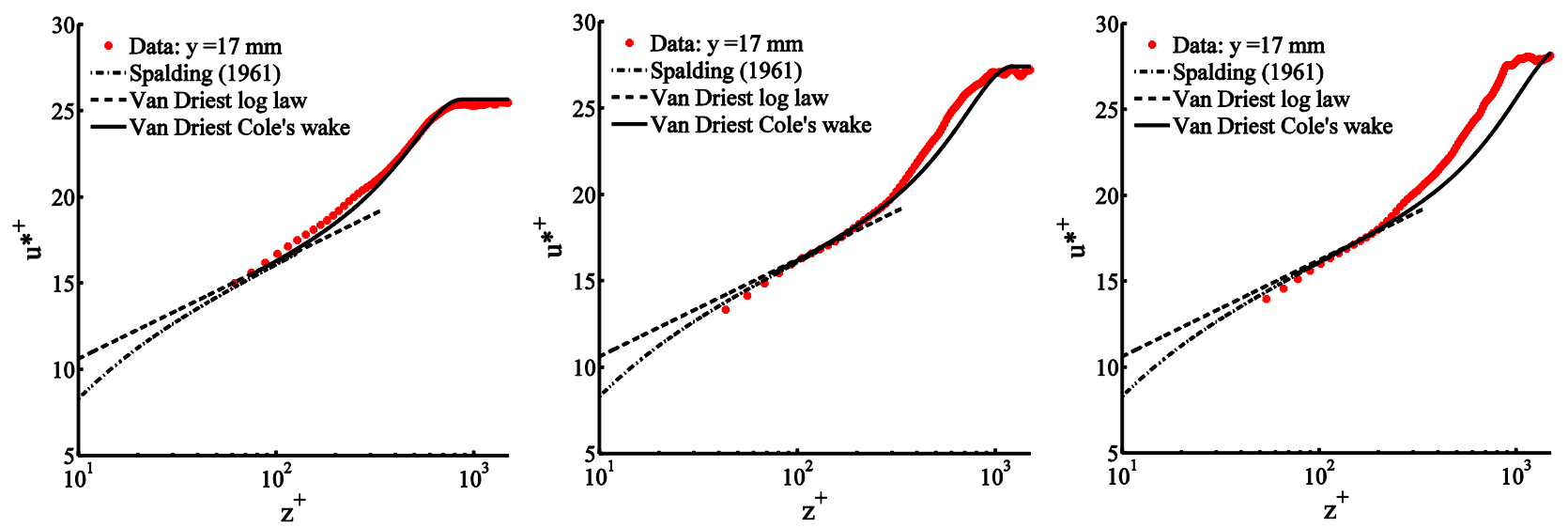


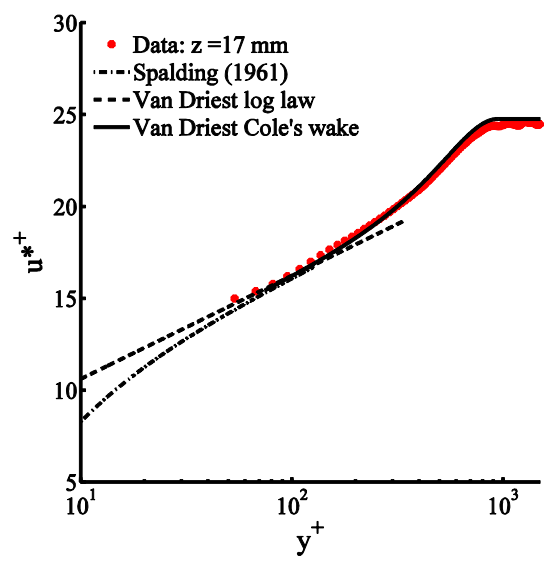

(a)

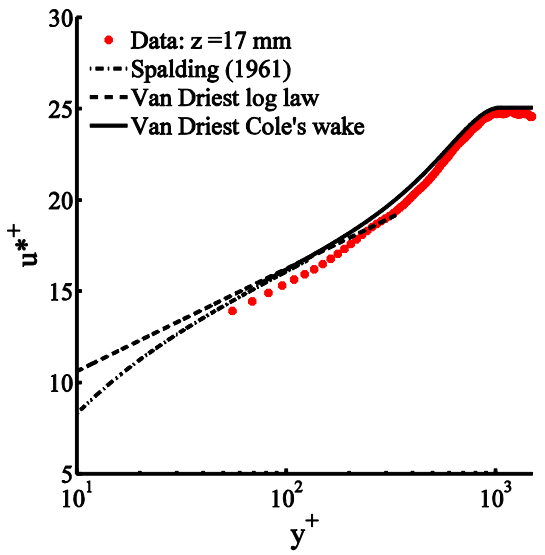

(b)

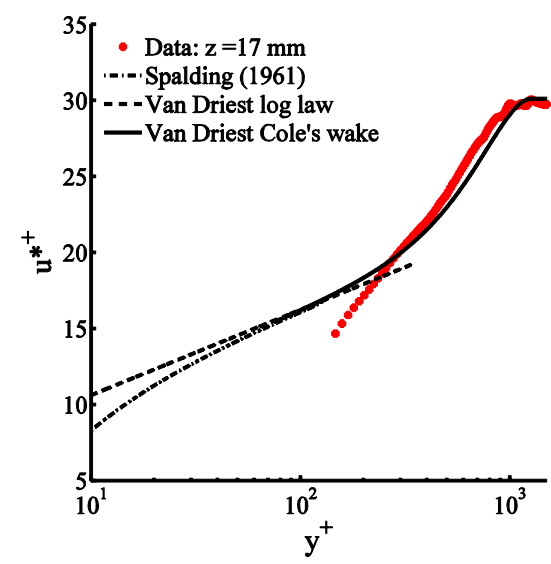

(c)

Figure 12. Velocity Scaling $17 \mathrm{~mm}$ away from the corner along bottom-wall (top) and along side-wall (bottom) at (a) $x=-100 \mathrm{~mm}$, (b) $x=-50 \mathrm{~mm}$, (c) $x=0 \mathrm{~mm}$
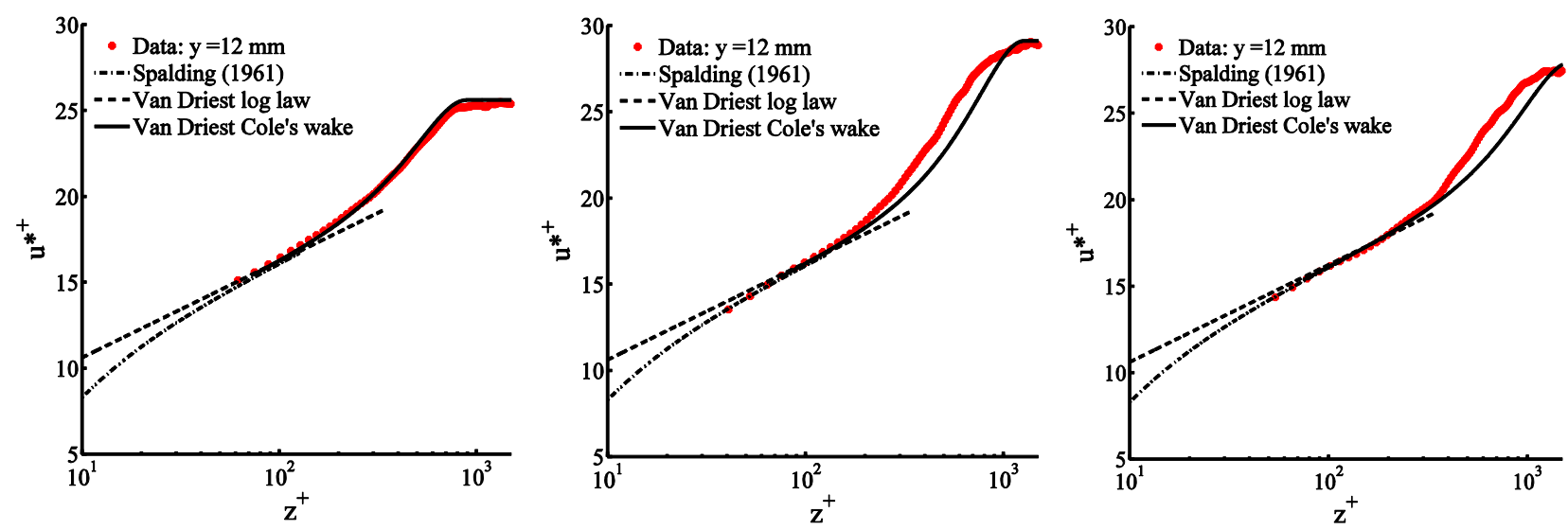

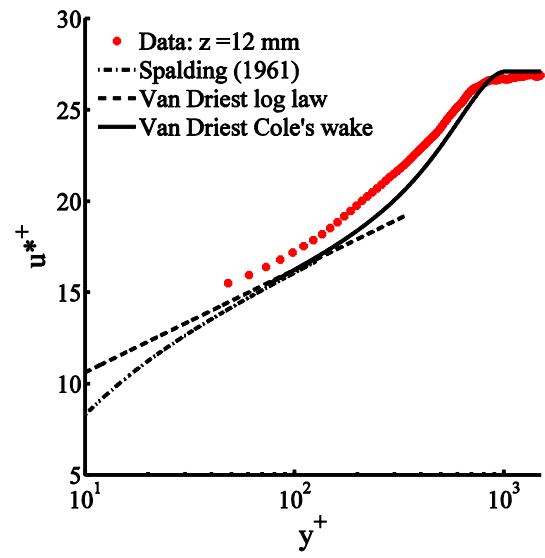

(a)

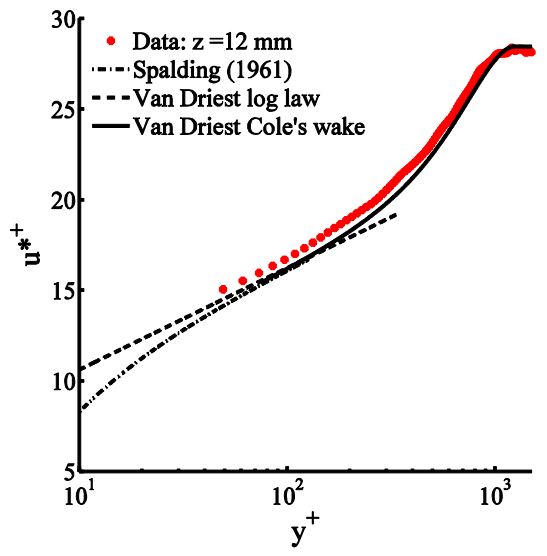

(b)

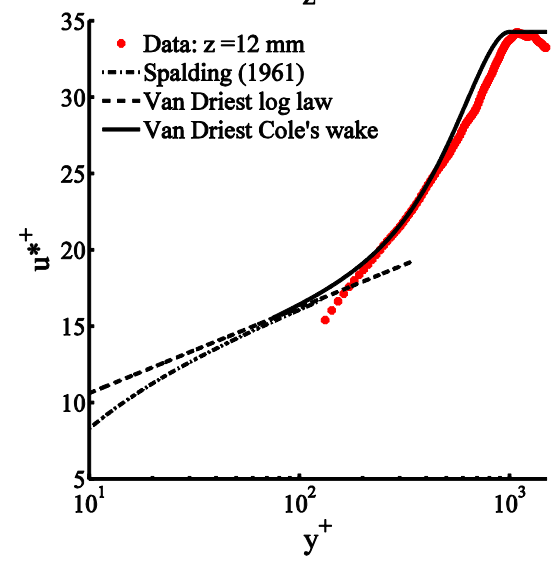

(c)

Figure 13. Velocity Scaling $12 \mathrm{~mm}$ away from the corner along bottom-wall (top) and along side-wall (bottom) at (a) $x=-100 \mathrm{~mm}$, (b) $x=-50 \mathrm{~mm}$, (c) $x=0 \mathrm{~mm}$ 


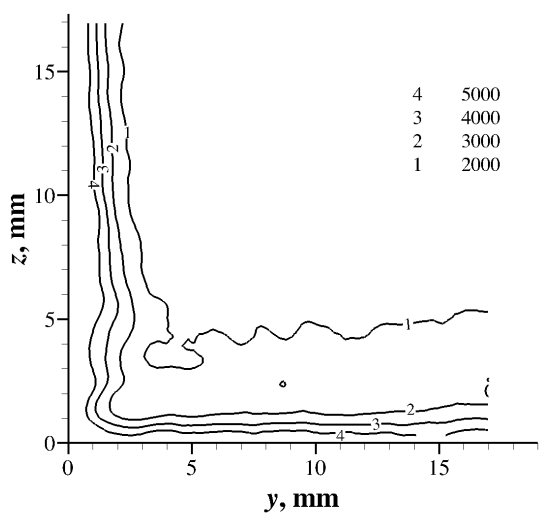

(a)

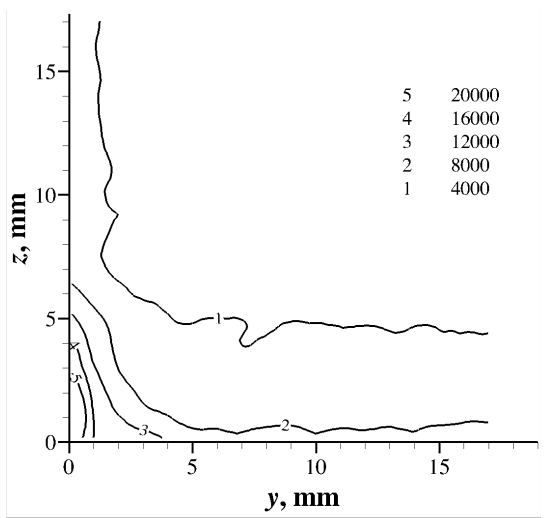

(b)

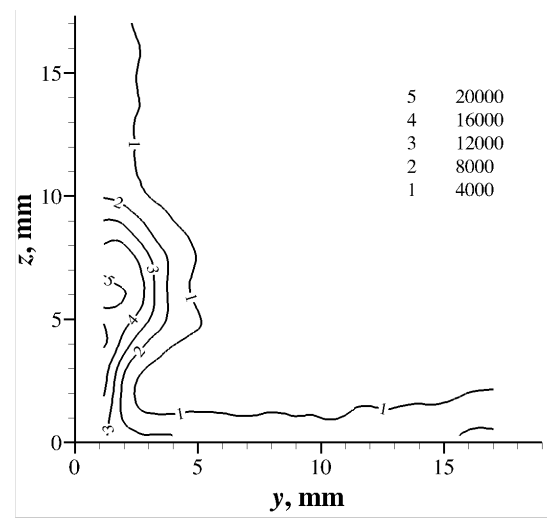

(c)

Figure 14. Multiple iso-contour levels (indicated in legend) of Reynolds stress $\overline{v^{\prime 2}}\left(\mathrm{~m}^{2} / \mathrm{s}^{2}\right)$ distribution at (a) $x$ $=-100 \mathrm{~mm}$, (b) $x=-50 \mathrm{~mm}$ and (c) $x=0 \mathrm{~mm}$

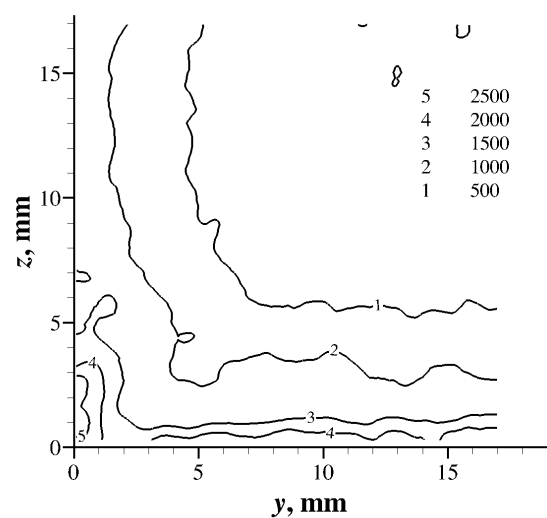

(a)

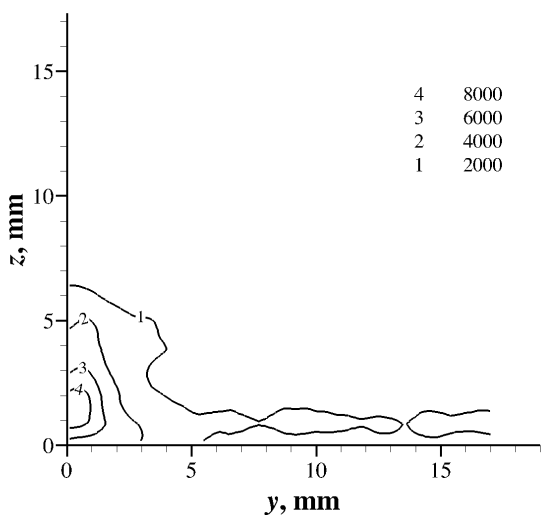

(b)

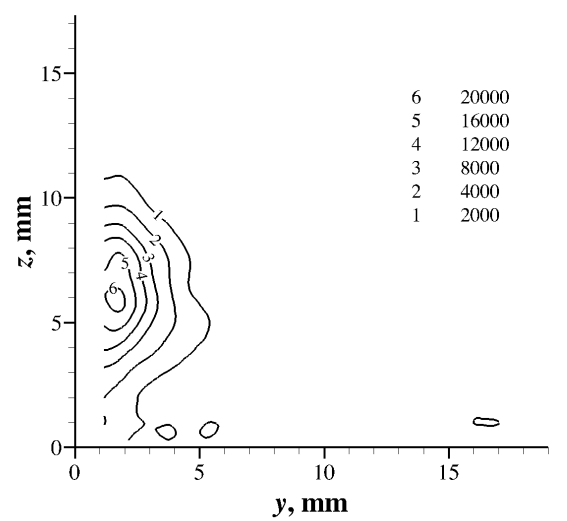

(c)

Figure 15. Multiple iso-contour levels (indicated in legend) of Reynolds stress $\overline{w^{\prime 2}}\left(\mathrm{~m}^{2} / \mathrm{s}^{2}\right)$ distribution at (a) $x$ $=-100 \mathrm{~mm}$, (b) $x=-50 \mathrm{~mm}$ and (c) $x=0 \mathrm{~mm}$

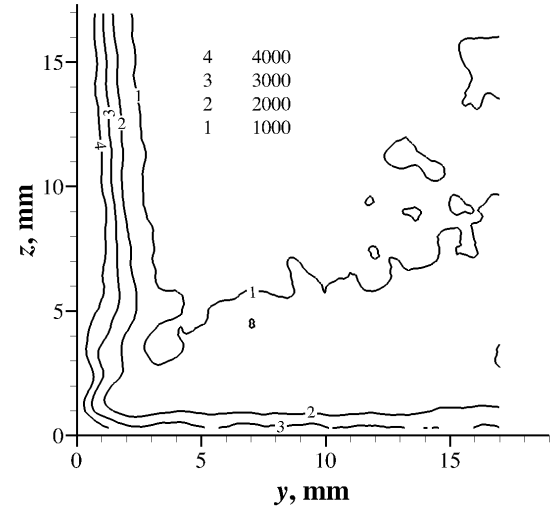

(a)

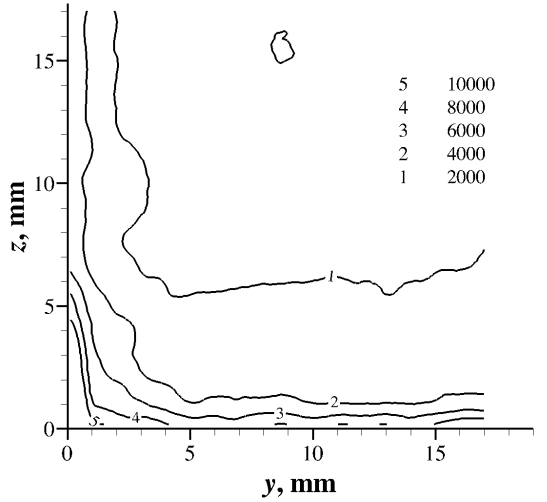

(b)

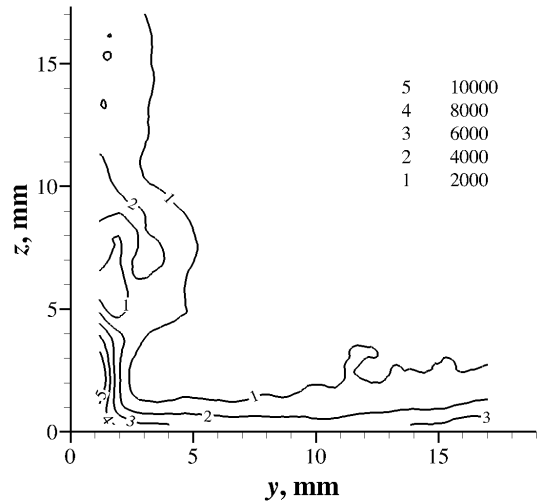

(c)

Figure 16. Multiple iso-contour levels (indicated in legend) of $\overline{v^{\prime 2}}-\overline{w^{\prime 2}}\left(\mathrm{~m}^{2} / \mathrm{s}^{2}\right)$ distribution at (a) $x=-100$ $\mathrm{mm}$, (b) $x=\mathbf{- 5 0} \mathrm{mm}$ and (c) $x=0 \mathrm{~mm}$. 


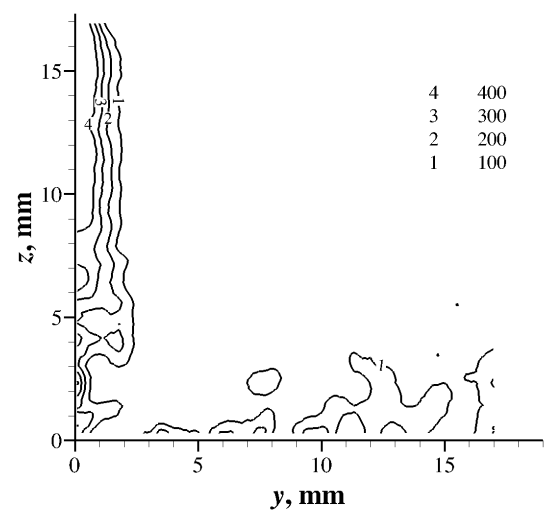

(a)

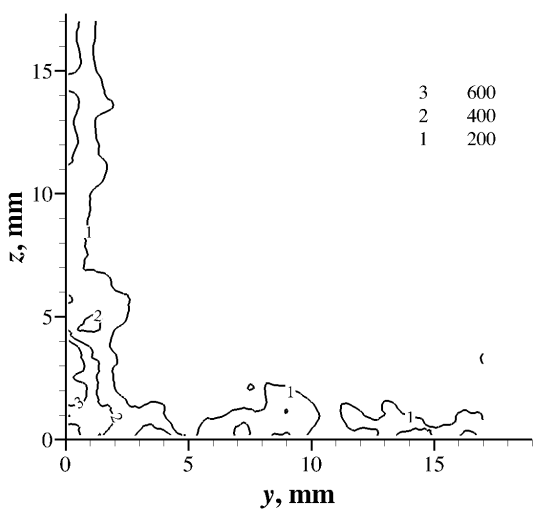

(b)

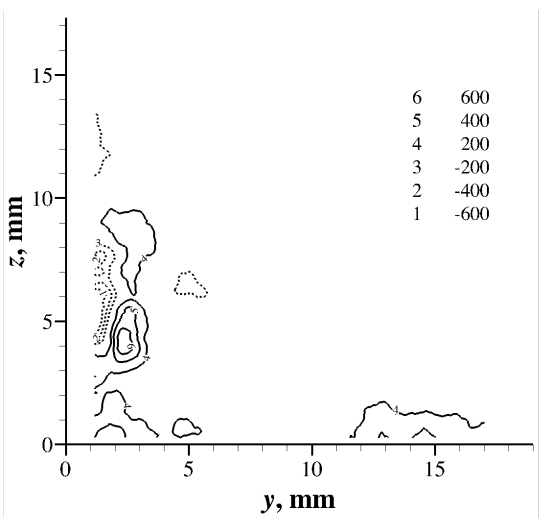

(c)

Figure 17. Multiple iso-contour levels (indicated in legend) of Reynolds stress $\overline{v^{\prime} w^{\prime}}$ distribution at (a) $x=-100$ $\mathrm{mm}$, (b) $x=-50 \mathrm{~mm}$ and (c) $x=0 \mathrm{~mm}$ 